\title{
Perspective
}

Actualité en histoire de l'art

\section{Les ivoires gothiques : foisonnement et renouveau des études}

Gothic Ivories: Flowering and Renewal of Studies

Gotische Elfenbeinarbeiten: Blütezeit und Neubetrachtung der Forschung

Gli avori gotici: proliferazione e rinnovamento degli studi

Los marfiles góticos: profusión y renovación de sus estudios

\section{Michele Tomasi}

\section{OpenEdition}

\section{Journals}

Édition électronique

URL : https://journals.openedition.org/perspective/7301

DOI : 10.4000/perspective.7301

ISSN : 2269-7721

Éditeur

Institut national d'histoire de l'art

Édition imprimée

Date de publication : 30 juin 2017

Pagination : 153-158

ISBN : 9782917902387

ISSN : 1777-7852

\section{Référence électronique}

Michele Tomasi, «Les ivoires gothiques : foisonnement et renouveau des études », Perspective [En ligne], 1 | 2017, mis en ligne le 31 décembre 2017, consulté le 16 janvier 2023. URL : http:// journals.openedition.org/perspective/7301; DOI : https://doi.org/10.4000/perspective.7301 


\section{Les ivoires gothiques : foisonnement et renouveau des études}

\section{Michele Tomasi}

\author{
- DAVIES, GUÉRIN, 2014 : Glyn Davies, Sarah M. Guérin \\ (dir.), New Work on Old Bones: Recent Studies in Gothic Ivories, \\ numéro thématique de la revue Sculpture Journal, n 23-1, \\ 2014, 115 p. \\ - GUÉRIN, 2015 : Sarah M. Guérin (dir.), Gothic Ivories. \\ Calouste Gulbenkian Collection, Londres, Scala, 2015, 128 p. \\ - Williamson, Davies, 2014 : Paul Williamson, Glyn \\ Davies, Medieval Ivory Carvings, 1200-1550, 2 vol., Londres, \\ V\&A Publishing, 2014, 928 p.
}

Depuis 2015, une base de données en ligne du Courtauld Institute of Art recense plus de cinq mille objets en ivoire (ou en os) sculptés en Europe entre 1200 et 1530 environ, en incluant les imitations ou falsifications et en excluant les produits issus de l'atelier des Embriachi et ceux qui leurs sont apparentés ${ }^{1}$. Lancé en 2008, le projet ayant abouti à cette base, dont John Lowden a été l'initiateur et Catherine Yvard la responsable, a encore stimulé les études sur les ivoires gothiques, qui faisaient déjà preuve d'un remarquable dynamisme depuis la fin des années 1990, sur la lancée d'un renouveau amorcé à la fin des années $1970^{2}$. Deux colloques organisés en lien avec le projet ont eu lieu à Londres en 2012 et en 2014 : certaines communications du premier ont été publiées dans un numéro thématique du Sculpture Journal en 2014, alors que plusieurs interventions du second viennent de paraître dans un ouvrage en ligne³.

\section{L'importance reconnue des ivoires gothiques}

L'ampleur même de la base du Courtauld témoigne de l'importance que les ivoires acquirent pendant les derniers siècles du Moyen Âge. Leur diffusion géographique et chronologique, ainsi que leur variété typologique et iconographique, font de ces œuvres des documents de premier ordre pour comprendre la culture artistique, la vie spirituelle et les mentalités, dans les domaines religieux comme profane. L'étude de ce matériau est pourtant restée longtemps confinée à un petit milieu de spécialistes, en raison d'un désintérêt généralisé à l'égard des arts considérés comme mineurs, mais aussi à cause d'une certaine défiance face à des objets qui posaient et posent des questions délicates de datation et de localisation, voire même d'authenticité.

Les équilibres ont pourtant changé, comme le montrent quelques publications récentes et importantes. L'aspect matériel de ces ouvrages en est déjà la preuve : nous avons affaire à des livres de grand format, où les sculptures et les reliefs en ivoire sont illustrés par des photos en couleurs, souvent sous plusieurs points de vue ou avec des détails saisissants. Bien que l'illustration ait été très soignée dans les trois cas ici pris en compte, il faut reconnaître que l'appareil iconographique du nouveau catalogue des ivoires gothiques du Victoria and Albert Museum est particulièrement somptueux. Les photos d'ensemble y reproduisent les objets à leur taille réelle, ou presque (dans ce cas, on indique précisément à quelle échelle ils sont réduits) - un choix que l'on ne peut assez louer.

La collection du V\&A est l'une des plus significatives au monde. Ce catalogue vient compléter la triade qui présente l'ensemble des ivoires du musée londonien depuis la période paléochrétienne jusqu'à l'époque moderne ${ }^{4}$. Il remplace ainsi la dernière vue d'ensemble qui en avait été offerte par Margaret Longhurst il y a bientôt un siècle ${ }^{5}$. Paul Williamson, conservateur en charge des ivoires du musée depuis la fin des années 1970, a été parfaitement secondé dans l'étude de ces objets par Glyn Davies. Le résultat est admirable. Les deux volumes comprennent deux cent quatre-vingt-neuf notices, dont certaines concernent des objets acquis après la parution du catalogue de Longhurst, et qui étaient souvent inédits. C'est le cas, par exemple, des très nombreux coffrets des Embriachi offerts au V\&A par Walter Leo Hildburgh en 1950, et donc présentés ici pour la première fois $\left(\mathrm{n}^{\mathrm{os}} 262-263\right.$, 265, 267, 270, 273-274, 279 et 281). Aucune autre institution ne conserve un ensemble aussi large et varié d'objets fabriqués par les Embriachi ou apparentés, et la partie consacrée à l'analyse de ces pièces, due à Glyn Davies, constitue une contribution majeure à leur connaissance. Certains coffrets jusqu'ici inédits ouvrent, par ailleurs, des pistes nouvelles : le $\mathrm{n}^{\circ} 267$ présente sous les scènes des traces d'inscriptions, peu lisibles. Il serait intéressant de les déchiffrer, car elles s'insèrent aisément dans la tradition des tituli, commençant toujours par le mot Chome ( Voici comment..." ; 
1. Atelier de Baldassarre

Ubriachi,

"Chome Paris inchoro[na] », détail d'un Coffret octogonal avec l'histoire de Pâris et figures de vertus, v. 1390-1410, Londres, Victoria and Albert Museum.

2. Norvège (probablement Trondheim), Crosse, fin du XIV siècle, Londres, Victoria and Albert Museum.

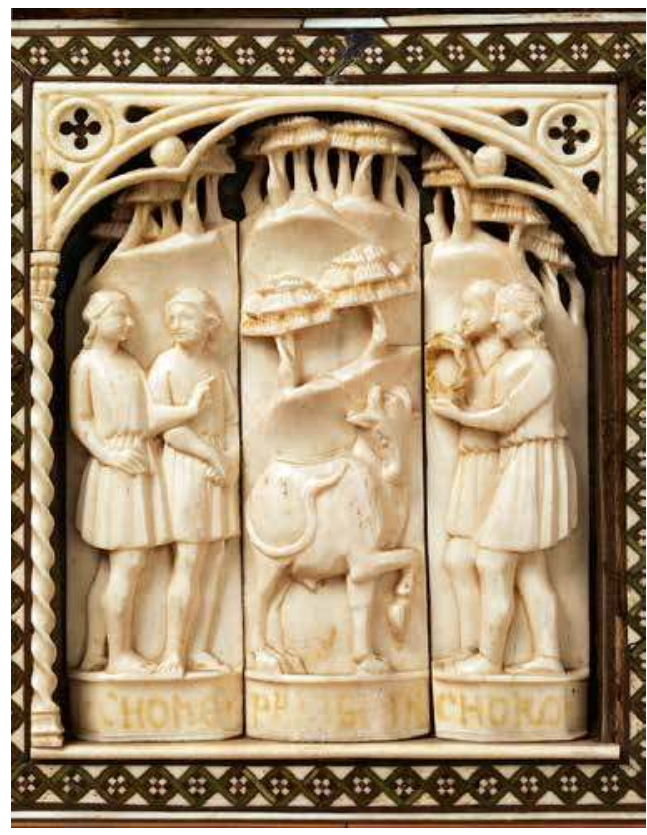

fig. 1). Elles pourraient peut-être nous renseigner sur les sources littéraires précises des scènes, et plus certainement sur les modalités de lecture et d'interprétation des histoires sculptées.

À côté des inédits, on trouve bien sûr tant les pièces célèbres, comme le diptyque Salting $\left(n^{\circ} 79\right)$ ou le livret dévotionnel colonais $\left(n^{\circ} 121\right)$, que des pièces moins connues et néanmoins frappantes, comme cette crosse norvégienne de la fin du XIV $\mathrm{XI}^{\mathrm{e}}$ siècle $\left(\mathrm{n}^{\circ} 153\right.$; fig. 2$)$. Les notices ne fournissent pas seulement un rigoureux état des connaissances, mais livrent souvent le résultat de recherches nouvelles, et deviennent parfois de courts essais qui constituent la meilleure synthèse sur un sujet donné, comme pour ce groupe de magnifiques reliefs ajourés et peints, sculptés à Paris au début du XV $\mathrm{X}^{\mathrm{e}}$ siècle (n ${ }^{\text {os }}$ 157-159).

\section{L'attention au matériau}

Les analyses des auteurs s'appuient sur un examen minutieux de chaque pièce. Cette démarche peut sembler anodine, mais elle produit souvent d'importants résultats. Tous les objets ont été mesurés à nouveau, ce qui a permis de corriger les dimensions fausses parfois données par le passé ; c'est ainsi que l'on a pu relier des pièces du musée à d'autres conservées ailleurs avec lesquelles elles formaient à l'origine une unité : un volet de diptyque ( $\left.\mathrm{n}^{\circ} 104\right)$ auquel on peut désormais associer son compagnon, conservé à l'Ashmolean

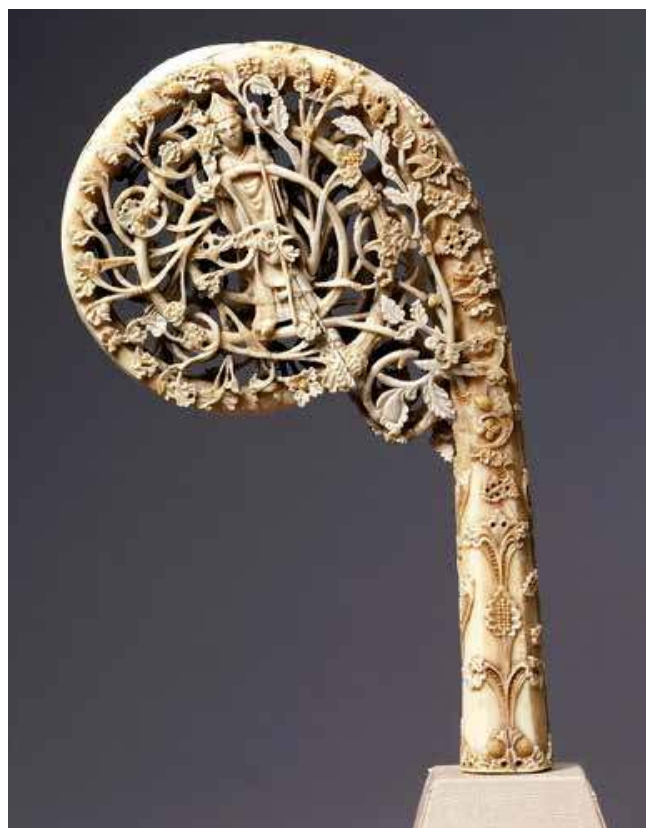

Museum d'Oxford (inv. WA 1908.185), retrouve ainsi son histoire, puisque l'on peut retracer la provenance de celui d'Oxford jusqu'à la seconde moitié du XVII ${ }^{\mathrm{e}}$ siècle ${ }^{6}$. Plus intéressantes encore sont les suites de l'examen attentif des charnières qui relient des volets. Les auteurs ont observé l'usage de deux autres types de charnières, différents de ceux que l'on connaissait déjà pour l'époque gothique ${ }^{7}$. L'un de ces deux " nouveaux " types était couramment utilisé pour les diptyques consulaires de l'antiquité tardive, ce qui pose la question de la connaissance que l'on avait de ceux-ci à la fin du Moyen Âge ( $\left.n^{\circ} 79\right)$. Une catégorie d'objets qui n'avait jamais été envisagée comme un modèle possible pour les ivoires gothiques devra désormais être prise en considération dans ce contexte.

L'attention accrue à la matérialité des sculptures en ivoire est l'un des points forts des recherches récentes. Sarah Guérin sait habilement analyser et expliquer les contraintes techniques de la fabrication. Elle le prouve encore une fois dans les notices de son catalogue d'une collection d'ivoires gothiques, restreinte mais de très haute qualité, celle réunie par Calouste Goulbenkian (1869-1955) à partir de 1914, et présentée dans le célèbre musée qui porte son nom à Lisbonne. Dans les onze notices très développées qu'elle consacre à ces œuvres, son attention se porte constamment sur la manière dont les plaques en ivoire ont été 
extraites de la défense, et sur les solutions trouvées par les ivoiriers afin d'exploiter au mieux ce matériau rare et précieux. Le triptyque avec la vie, la mort et le triomphe de la Vierge $\left(\mathrm{n}^{\circ} 7\right)$, dont la pointe du gâble central, perdue, était sculptée à part et autrefois attachée par des chevilles et de la colle, en est un bon exemple (fig. 3). Ce système d'assemblage se retrouve sur quelques autres objets récemment étudiés par Adam Harris Levine ${ }^{8}$. Notre compréhension de la réalité concrète du travail dans l'atelier progresse ainsi grâce à une démarche qui avait été testée avec succès et de manière pionnière sur les ivoires byzantins ${ }^{9}$. Un même esprit est à l'œuvre dans l'attention portée à la polychromie ${ }^{10}$

Dans le monde anglo-saxon, l'étude des ivoires s'appuie aussi, depuis une quinzaine d'années et de plus en plus souvent, sur des analyses scientifiques, et notamment sur la datation au carbone 14. Des prélèvements ont été effectués sur dix-sept objets du V\&A (WILLIAMSON, DAVIES, 2014, vol. II, p. 876-879). Le système permet de dater approximativement la mort de l'éléphant, et de connaître donc la date après laquelle la défense devient disponible pour le sculpteur. Le résultat de telles analyses n'est que l'une des données à prendre en compte, avec toutes les autres : la typologie, l'iconographie, le style, la provenance ${ }^{11} \ldots$ Quand le résultat de l'analyse s'accorde avec les autres éléments, il peut apporter une confirmation décisive. Lorsqu'en revanche il y a divergence, les problèmes surgissent. Parfois le carbone 14 indique que la défense serait d'un ou deux siècles plus ancienne que la date présumée pour la sculpture : doit-on alors imaginer que le matériau brut, pourtant rare et précieux, ait été stocké pendant si longtemps ${ }^{12}$ ? Ou alors que les intermédiaires commercialisaient aussi des défenses trouvées très longtemps après la mort des animaux ${ }^{13}$ ? Mais alors on peut tout aussi bien imaginer que de l'ivoire ancien était disponible pour des sculpteurs travaillant en style ou des faussaires, une hypothèse qui est en effet envisagée pour quatre objets du V\&A, dont on n'exclut pas qu'ils puissent être des œuvres du XIX ${ }^{\mathrm{e}}$ siècle $\left(\mathrm{n}^{\text {os }} 19\right.$,

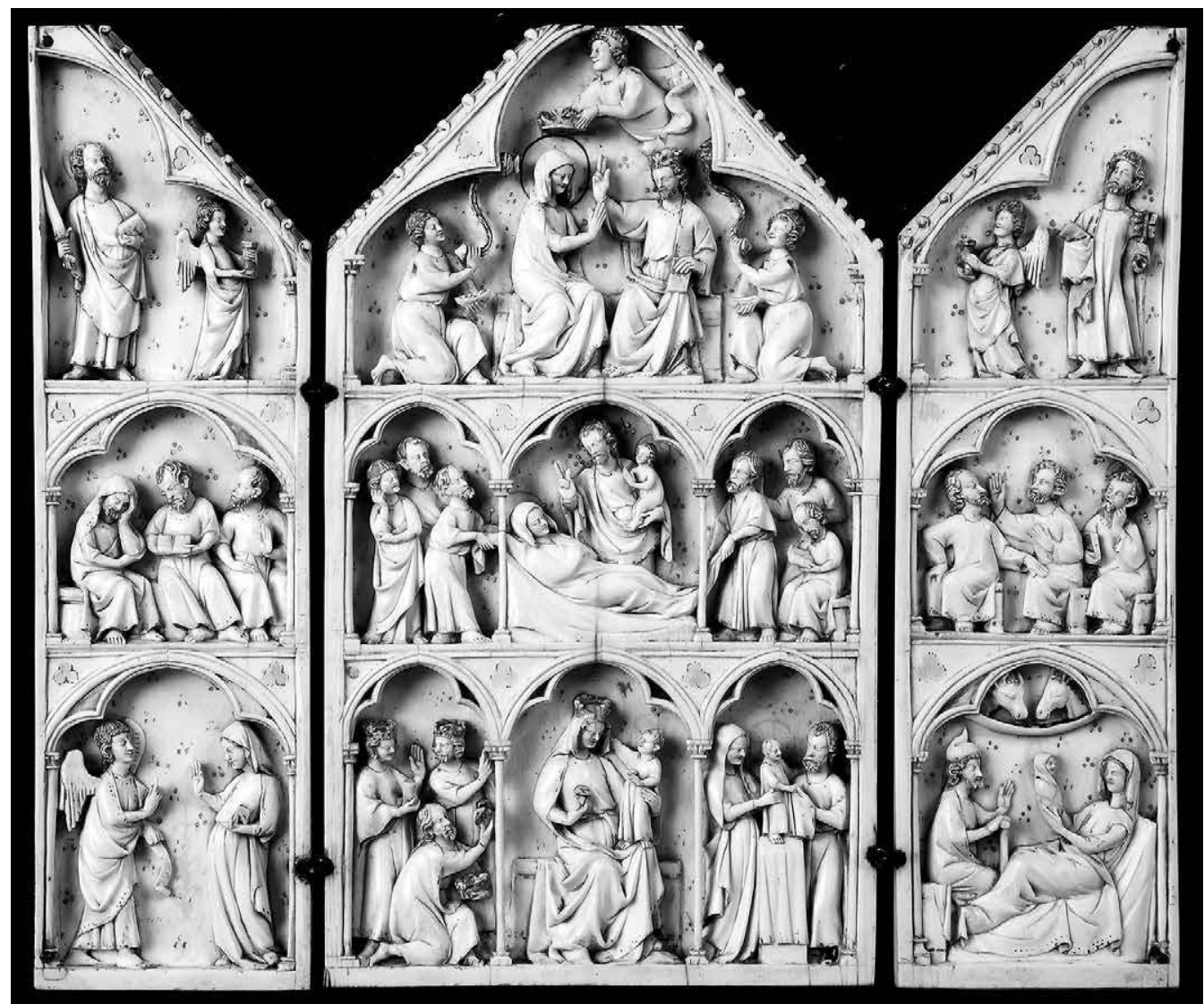

3. Maître de la mort de la Vierge (Paris), Triptyque avec scènes de la vie et de la mort de la Vierge, 1280-1300, Lisbonne, Museu Calouste Gulbenkian. 
4. France (Paris) ou Italie du Nord (Milan?), Diptyque avec scènes de la vie et du triomphe de la Vierge et de la Passion du Christ, v. 1414, Londres, Victoria and Albert Museum.
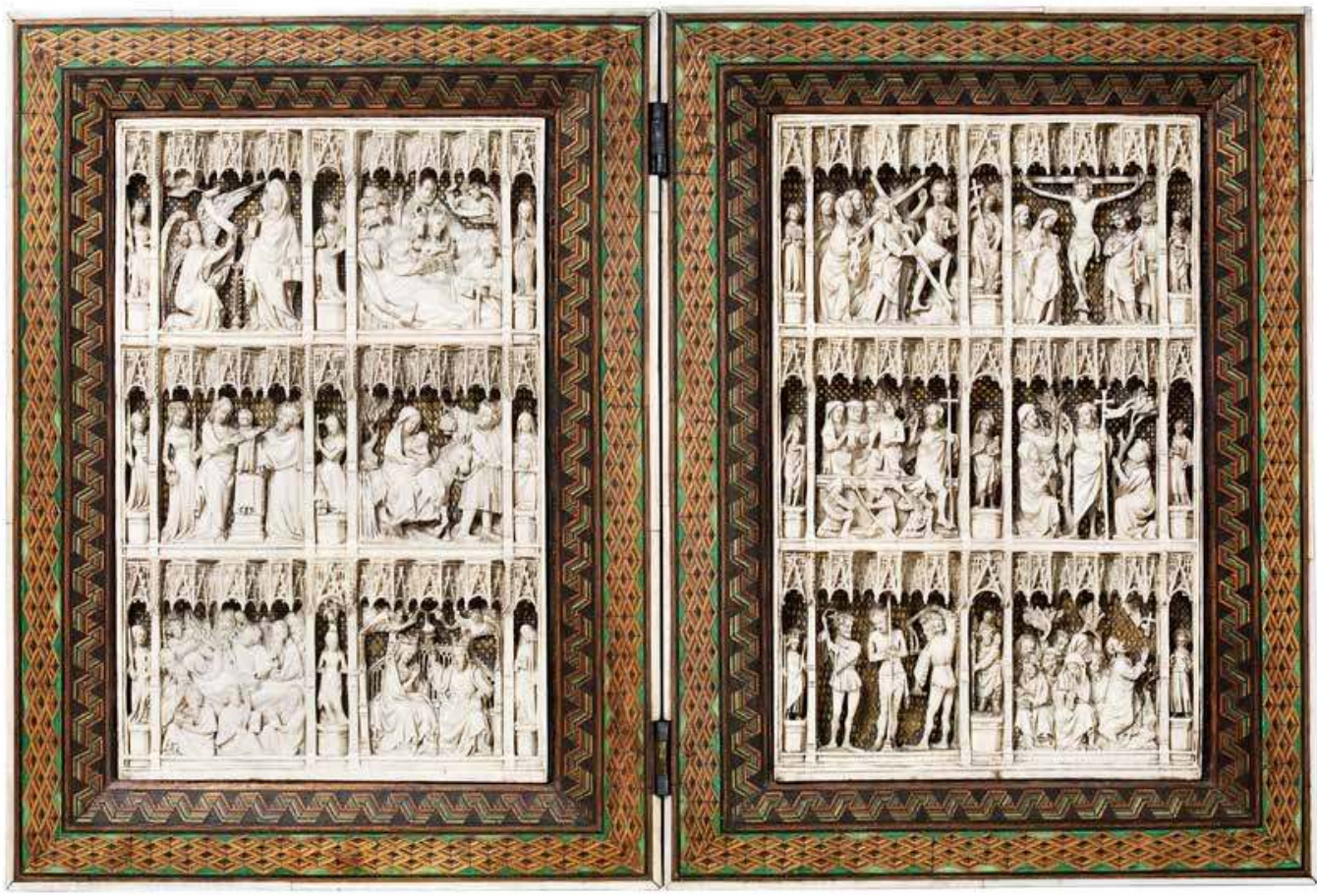

144, 154 et 190). La question de la fiabilité du radiocarbone est d'autant plus brûlante que le statut de quelques pièces cruciales en dépend : $c^{\prime}$ est le cas d'un impressionnant et très singulier triptyque de la collection Thomson qui est soit un faux, soit la pièce maîtresse de l'un des groupes d'ivoires les plus beaux et les plus importants produits à Paris autour de $1300^{14}$. Néanmoins, le problème reste épineux puisque des voix s'élèvent parfois pour poser le problème de la pertinence de ce type d'analyse pour les ivoires ${ }^{15} \ldots$

\section{Questions d'attribution, de datation, de localisation}

Sur la question des falsifications, les études récentes ont permis de véritables avancées. En 1969, un article notoire de Jaap Leeuwemberg avait jeté la suspicion sur plus d'une centaine de pièces gothiques, d'une manière assez cavalière $^{16}$. Plusieurs objets condamnés par le savant néerlandais sont aujourd'hui réhabilités, sur la base d'un examen plus soigné de leur matérialité, de leur provenance, de leur iconographie : cela vaut notamment pour le fragment d'une Cène $\left(\mathrm{n}^{\circ} 39\right)$ ou un panneau de coffret ajouré $\left(\mathrm{n}^{\circ} 231\right)$ du V\&A ${ }^{17}$. Des pièces appartenant aux mêmes groupes et conservées au Metropolitan Museum of Art de New York ont été récemment réétudiées par Charles Little qui parvient à des conclusions similaires ${ }^{18}$. Tout l'ensemble des ivoires ajourés avait par ailleurs déjà été justement défendu, et replacé dans son contexte, qui est celui du Nord de la France dans le troisième quart du XIV siècle, par Benedetta Chiesi ${ }^{19}$. Un diptyque du V\&A (n $\left.{ }^{\circ} 112\right)$ est aussi reconnu comme une œuvre authentique, du début du $\mathrm{XV}^{\mathrm{e}}$ siècle, en prenant en compte son étui original en cuir, qui porte les armoiries de Bureau Boucher, maître des requêtes de Charles VI, et de son épouse (fig. 4). Paul Williamson suggère que le diptyque pourrait avoir été fabriqué à Paris, ou bien encore à Milan, en partie en raison du cadre marqueté de l'œuvre. Dans ce cas précis, il faudrait vérifier la biographie des commanditaires pour étayer ou rejeter l'hypothèse d'une fabrication milanaise, qui paraît a priori la moins probable. Plus largement, plusieurs pièces du V\&A sont attribuées de manière convaincante à l'Italie du Nord, et avec circonspection à la Lombardie ou à Venise (n ${ }^{\text {os }} 207,210-214$ et 221-223). L'organisation du catalogue, qui sépare les objets religieux des objets profanes, et regroupe les pièces selon la typologie, ne permet pas de saisir d'emblée l'ampleur de cet ensemble italien; mais si on y prête attention, il devient clair qu'il est 

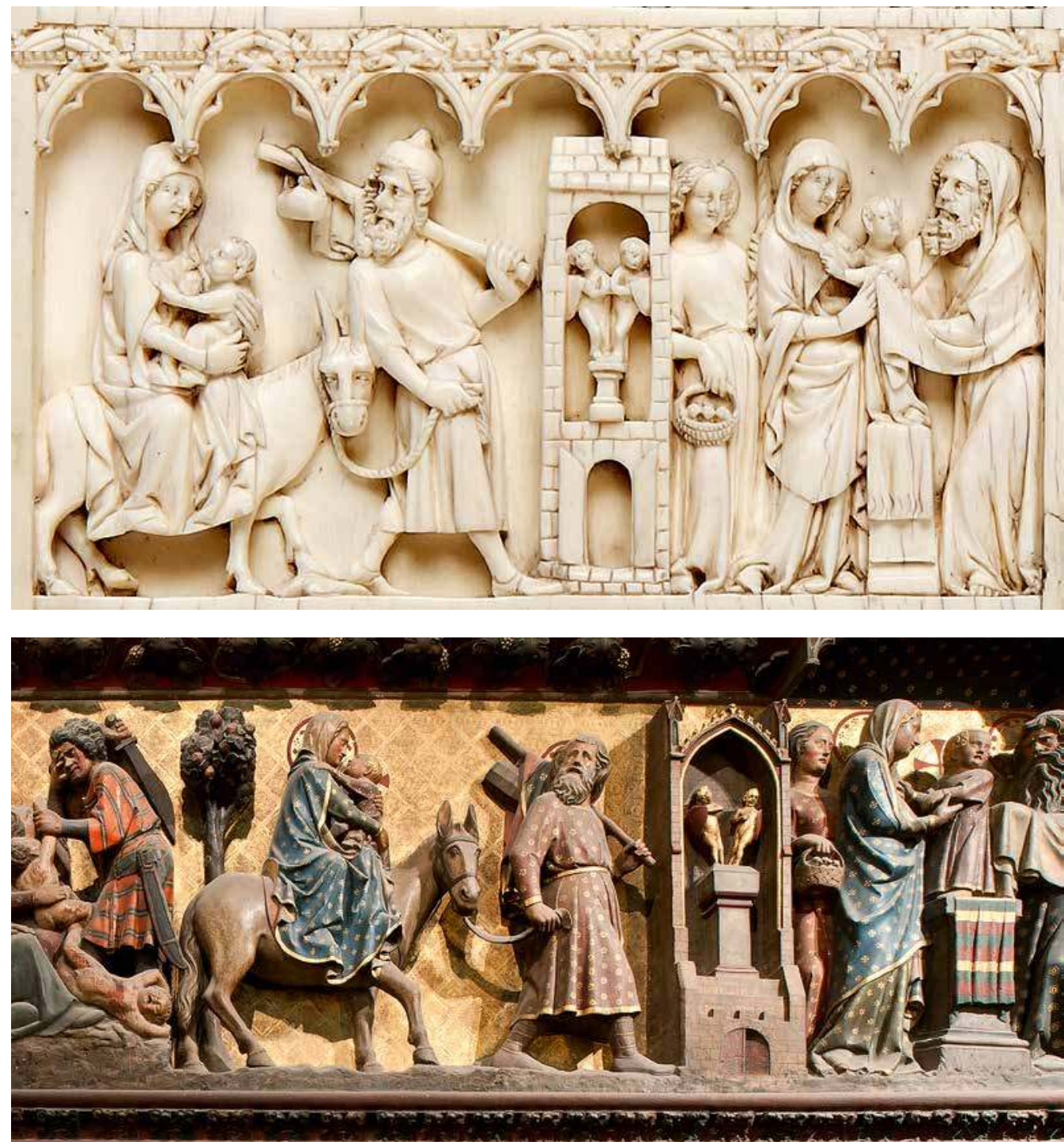

5a. Fuite en

Égypte et Présentation au Temple, détail du Diptyque de la Passion, Paris, v. 1350-1375, Lisbonne, Museu Calouste Gulbenkian.

5b. Pierre de Chelles, Fuite en Égypte et Présentation au Temple, détail de la clôture nord du chœur, 1305-1315, Paris, cathédrale Notre-Dame. souhaitable de reprendre tout le dossier pour comprendre si Venise et/ou Milan ont joué un rôle dans l'ivoirerie gothique et lequel ${ }^{20}$.

Les questions de datation et de localisation occuperont encore longtemps les études sur les ivoires $^{21}$. Dans ce domaine, le décloisonnement de la recherche a été très bénéfique, car nombre d'objets en ivoire ont pu être mieux cernés en les comparant à des créations dans d'autres arts - sculpture monumentale, enluminure, vitrail, orfèvrerie... Le catalogue du V\&A en offre de nombreuses preuves, tout comme celui rédigé par Sarah Guérin : qu'il suffise de mentionner le rapport très précis qu'elle a repéré entre un grand diptyque de la Passion $\left(\mathrm{n}^{\circ} 4\right)$ et la clôture du chœur de Notre-Dame à Paris, qui démontre que l'ivoirier connaissait bien l'œuvre monumentale (fig. 5a-b). Dans le catalogue de la collection Gulbenkian, les images de comparaison sont très nombreuses et soutiennent efficacement la démonstration - comme pour ce tabernacle attribué à Cologne $\left(n^{\circ} 6\right)$, entre autres sur la base du style des images peintes à l'extérieur de ses volets. Si le style et les compositions restent des données cruciales pour procéder à des attributions, les travaux récents prennent systématiquement en compte un éventail d'indices plus variés. C'est le cas pour une statuette de la Vierge et l'Enfant 
trônant de Londres $\left(\mathrm{n}^{\circ} 13\right)$ qui fait partie d'un groupe d'œuvres considéré comme anglais depuis les années 1980. Paul Williamson, qui trouve peu probants les rapprochements stylistiques avancés jusqu'ici, fait remarquer qu'aucune provenance ancienne ne vient étayer cette attribution, et que plusieurs des statuettes du même ensemble ont en revanche une provenance normande - et propose ainsi de situer dans cette région la création de ces pièces. Il sera intéressant de comparer ces ivoires avec la statuaire gothique normande pour vérifier si le style peut confirmer cette proposition.

\section{Des questions nouvelles}

Cette attention à la matérialité ou aux formes ne nuit pas à l'intérêt pour les contenus. Les ivoires gothiques font aussi désormais l'objet d'analyses iconographiques subtiles et complexes. Que l'on lise par exemple comment Sarah Guérin explique l'association de l'Adoration des Mages et de la Crucifixion, récurrente sur les diptyques, comme un support pour la méditation et la prière $\left(n^{\circ} 1\right)$. De manière analogue, Paula Mae Carns donne un nouvel exemple de son habileté dans l'étude de l'iconographie profane et de ses rapports complexes avec les textes, en décryptant finement le discours que construisent deux coffrets avec des scènes de la folie de Tristan ${ }^{22}$.

Signe de la maturité atteinte dans ce domaine de recherches, on notera pour terminer que l'on s'intéresse maintenant beaucoup aussi à l'histoire du collectionnisme et de l'étude des ivoires. Émile van Binnebeke relate par exemple l'histoire de la fabrication de moulages d'ivoires dans l'atelier spécialisé des Musées Royaux d'Art et d'Histoire à Bruxelles ${ }^{23}$, tandis que Sarah Guérin replace les achats d'ivoires gothiques par Calouste Gulbenkian dans le contexte plus large de l'histoire du goût et du collectionnisme dans la première moitié du XXe siècle (GUÉRIN, 2015, p. 16-35). De manière analogue, on a récemment éclairé la figure de Thomas Gambier Parry (1816-1888), notamment en tant que collectionneur d'ivoires ${ }^{24}$, et on a mis en valeur les deux cent soixante-cinq photographies de moulages d'ivoires prises autour de 1850 par John Brampton Philpot, un photographe anglais installé à Florence ${ }^{25}$. Comme toujours lorsque l'on approche le Moyen Âge, il est indispensable de s'interroger sur l'histoire de sa réception si l'on souhaite en avoir une véritable compréhension. Les spécialistes des ivoires gothiques en sont aussi conscients, et contribuent à éclairer les mécanismes par lesquels les œuvres qui ont survécu ont été préservées en raison des choix faits autrefois, des choix qui ont déterminé les corpora sur la base desquels nous construisons nos réflexions.

Michele Tomasi, université de Lausanne michele.tomasi@unil.ch 
1. Www.gothicivories.courtauld.ac.uk (consulté le 20 décembre 2016). Voir Catherine Yvard, "The Gothic Ivories Project at the Courtauld Institute of Art ", dans DAVIES, GUÉRIN, 2014, p. 98-100. La base n'est pourtant pas complète : elle n'inclut pas, par exemple, les pièces du Museu Calouste Gulbenkian de Lisbonne. L'atelier dit des Embriachi, qui appartint au marchand florentin Baldassarre Ubriachi, fut actif à Florence puis à Venise entre 1370 environ et 1430 environ. Il produisit des centaines de coffrets, triptyques et autres objets avec des reliefs en os appliqués sur une structure en bois ornée de marqueterie; en raison de l'abondance même de cette production, une base de données consacrée uniquement à cet atelier aurait été nécessaire.

2. Pour un bilan des recherches jusqu'en 1997, voir Michele Tomasi, "Rassegna - Avori gotici. Studi e mostre recenti ", dans Annali della Scuola Normale Superiore di Pisa. Classe di Lettere e Filosofia, s. IV, n II-1, 1997 (1999), p. 215-232. Pour les vingt dernières années, voir Danielle Gaborit-Chopin, " Gothic Ivories: Realities and Prospects ", dans Colum Hourihane (dir.), Gothic Art $\theta$ Thought in the Later Medieval Period: Essays in Honor of Willibald Sauerländer, Princeton, 2011, p. 156-175, et Glyn Davies, Sarah M. Guérin, "Introduction ", dans DAVIES, GUÉRIN, 2014, p. 7-12.

3. Catherine Yvard (dir.), Gothic Ivories: Content and Context, Londres, 2017.

4. Paul Williamson, Medieval Ivory Carvings. Early Christian to Romanesque, Londres, 2010 ; Marjorie Trusted, Baroque and Later Ivories, Londres, 2013.

5. Margaret H. Longhurst, Catalogue of the Carvings in Ivory, Victoria and Albert Museum, Londres, 1927-1929, 2 vol.

6. La collection d'ivoires de l'Ashmolean Museum vient aussi de faire l'objet d'un superbe catalogue scientifique : Jeremy Warren, Medieval and Renaissance Sculpture. A Catalogue of the Collection in the Ashmolean Museum, Oxford, vol. II, Sculptures in Stone, Clay, Ivory, Bone and Wood, Oxford, 2014.

7. Voir le compte-rendu, par Sarah M. Guérin, de WILLIAMSON, DAVIES, 2014, dans The Burlington Magazine, n ${ }^{\circ}$ CLVI, 2014, p. 757-758, ici p. 758.

8. Adam Harris Levine, "Putting Together the Pieces: Composite Techniques in Gothic Ivories ", dans DAVIES, GUÉRIN, 2014, p. 41-50.

9. Anthony Culter, The Hand of the Master. Craftsmanship, Ivory and Society in Byzantium, Princeton, 1994 ; pour l'organisation du travail à l'époque gothique voir en dernier lieu la mise au point experte et nuancée de Danielle Gaborit-Chopin, "Documents et œuvres d'art : remarques sur quelques ivoires gothiques français ", dans Cahiers archéologiques, $\mathrm{n}^{\circ}$ 55, 2013-2014, p. 119-130.

10. Christine Kowalski, "Polychromy on Medieval Ivories from the LVR-LandesMuseum, Bonn ", dans DAVIES, GUÉRIN, 2014, p. 95-98; ici on trouvera aussi les références aux études fondatrices de Agnès Cascio, Juliette Levy et Danielle Gaborit-Chopin.

11. Voir John Lowden, Medieval and Later Ivories in the Courtauld Gallery: Complete Catalogue, avec une contribution d'Alexandra Gerstein, Londres, 2013, p. 18-19.
12. Katherine Eve Baker a en effet montré qu'à Paris, au XVI ${ }^{\mathrm{e}}$ siècle, de larges stocks d'ivoire pouvaient passer d'une génération de sculpteurs à l'autre, mais il est difficile de dire si ce constat peut être généralisé. Voir Katherine E. Baker, "Book Review: John Lowden, Medieval and Later Ivories in the Courtauld Gallery: Complete Catalogue, with a contribution by Alexandra Gerstein, London, Paul Holberton Publishing, 2013 ", dans Peregrinations. Journal of Medieval Art $\theta$ Architecture, $\mathrm{n}^{\circ} \mathrm{V}-1,2015$ (en ligne : http://peregrinations.kenyon.edu/vol5_1/ BakerPeregrinations51.pdf ; consulté le 20 décembre 2016) ; Eadem, " "La chambre aux dentz d'ivoire" : Late Flamboyant Ivory Carving in Paris and the Inventory of Chicart Bailly ", dans Yvard, 2017, citée n. 3.

13. Pour le commerce de l'ivoire à l'époque gothique, voir Danielle Gaborit-Chopin, " Le commerce de l'ivoire en Méditerranée durant le Moyen Âge ", dans Bulletin archéologique du CTHS, ${ }^{\circ}$ 34, 2008, p. 23-33; Sarah M. Guérin, "Avorio d'ogni ragione: the Supply of Elephant Ivory to Northern Europe in the Gothic Era ", dans Journal of Medieval History, $\mathrm{n}^{\circ}$ 36, 2010, p. 156-174.

14. L'authenticité du triptyque est défendue, sur la base de la datation au carbone 14 entre autres, dans la notice de l'excellent catalogue de la collection : John Cherry, John Lowden, Medieval Ivories and Works of Art. The Thomson Collection at the Art Gallery of Ontario, Toronto, 2008, n 28.

15. Francesca Dell'Acqua et alii (dir.), The Salerno Ivories: Objects, Histories, Contexts, Berlin, 2016, p. 10.

16. Jaap Leeuwemberg, "Early Nineteenth-Century Gothic Ivories ", dans Aachener Kunstblätter, nº 39, 1969 , p. 111-148. Pour une mise en garde précoce contre ses conclusions hâtives, voir Danielle Gaborit-Chopin, "Les ivoires gothiques : à propos d'un article récent ", dans Bulletin Monumental, $\mathrm{n}^{\circ} 128,1970$, p. 127-133.

17. Dans le catalogue londonien, les faux ne sont pas analysés dans une section à part, mais à la suite des originaux qu'ils imitent, ce qui permet des comparaisons éclairantes.

18. Charles T. Little, «The Art of Gothic Ivories: Studies at the Crossroads ", dans DAVIES, GUÉRIN, 2014, p. 13-29.

19. Benedetta Chiesi, Catalogo degli avori gotici del Museo Nazionale del Bargello, thèse, Università degli Studi di Firenze, 2011, p. 75-86 et $\mathrm{n}^{\text {os }}$ 28-29. L'auteure prépare, avec Danielle Gaborit-Chopin, le nouveau catalogue des ivoires de ce même musée.

20. L'organisation selon la typologie sépare d'autres objets qui sont étroitement apparentés entre eux, comme les deux diptyques et le triptyque appartenant au groupe dit de Soissons ( $\mathrm{n}^{\text {os }} 51,67-68$ ). Mais il est vrai qu'elle permet en revanche de saisir les transformations ou les permanences en fonction des types, et qu'un système de renvois internes très efficaces pallie ses défauts.

21. C'est l'une des raisons pour lesquelles les catalogues restent, dans ce domaine, des outils de recherche et de travail de première importance ; je me permets de signaler encore, dans cette catégorie, la parution récente de Simonetta Castronovo, Fabrizio Crivello, Michele Tomasi (dir.), Collezioni del Museo Civico d'Arte Antica di Torino: avori medievali, Savigliano (CN), 2016. 
22. Paula Mae Carns, "Playing the Fool: La Folie Tristan on two French Gothic Ivories ", dans DAVIES, GUÉRIN, 2014, p. 51-63.

23. Émile van Binnebeke, "Casting Ivories in Exchange? A Short History of the Brussels Atelier de Moulage ", dans DAVIES, GUÉRIN, 2014, p. 81-92.

24. Lowden, 2013, cité n. 11, p. 19-24; Alexandra Gerstein, "Thomas Gambier Parry: Collecting in the Gothic Revival ", ibid., p. 26-36.

25. Júlia Papp, Benedetta Chiesi (dir.), John Brampton Philpot's Photographs of Fictile Ivory, Budapest, 2016. 